\title{
Percutaneous CT-Guided Drainage of Gastric Leaks Post-Sleeve Gastrectomy
}

\author{
Sultan R. Alharbi \\ Department of Radiology and Medical Imaging, College of Medicine, King Saud University, Riyadh, Saudi Arabia \\ Email: drsultan000@gmail.com
}

How to cite this paper: Alharbi, S.R. (2020) Percutaneous CT-Guided Drainage of Gastric Leaks Post-Sleeve Gastrectomy. Open Journal of Radiology, 10, 1-8. https://doi.org/10.4236/ojrad.2020.101001

Received: January 22, 2020

Accepted: February 10, 2020

Published: February 13, 2020

Copyright $\odot 2020$ by author(s) and Scientific Research Publishing Inc. This work is licensed under the Creative Commons Attribution International License (CC BY 4.0).

http://creativecommons.org/licenses/by/4.0/

\section{(c) (i) Open Access}

\begin{abstract}
Objectives: The aim of this study was to assess the safety and efficacy of percutaneous CT-guided drainage of gastric leaks post sleeve gastrectomy. Methods: For this single-center retrospective study, we reviewed the clinical data of 78 patients ( 44 men and 34 women with an average age of $34.6 \pm 10.5$ years and a body mass index (BMI) of $\left.45 \mathrm{~kg} / \mathrm{m}^{2} \pm 3.2\right)$ that underwent percutaneous CT-guided drainage of gastric leaks due to sleeve gastrectomy from September 2011 to September 2018. The outcome measurements were technical and clinical success, complications, and the need for revisional surgery. Results: The technical success rate of drain insertion was $97.5 \%$ (76/78 patients). All of the patients (76/76 patients) exhibited early clinical and laboratory improvement, and no emergency surgery was required. However, six patients underwent revisional surgery after 3 - 5 months for non-healing gastric leaks/fistulas. One patient had a major complication of active bleeding due to arterial injury; this was managed by transcatheter coil embolization. All patients underwent endoluminal stent placement and received antimicrobial therapy and nutritional support. Conclusion: Percutaneous CT-guided drainage of gastric leaks after sleeve gastrectomy is a safe, effective, and minimally invasive alternative to surgery. This technique is in line with other conservative measures (endoluminal stent placement, antimicrobial therapy, and nutritional support), which heal most gastric leaks due to sleeve gastrectomy and prevent the need for revisional surgery.
\end{abstract}

\section{Keywords}

Percutaneous Drainage, CT-Guided Drainage, Sleeve Gastrectomy, Gastric Leak

\section{Introduction}

The reported rate of gastric leak after laparoscopic sleeve gastrectomy is $1 \%-3 \%$ 
for primary surgery and $7 \%-10 \%$ for secondary surgery [1] [2]. As more laparoscopic sleeve gastrectomies are being performed, more associated gastric leaks are being encountered. Gastric leak management is challenging and requires multidisciplinary teams and long hospital stays [3].

Although no established standard management protocol exists to date [4] [5], conservative minimal invasive management is encouraged over surgical revision, as surrounding inflammation and friable tissue decrease the success rate of surgical closure of leaks [4] [6]. Surgical management is suitable for unstable septic patients with early leaks [4] [5] [6], whereas conservative management is suitable for hemodynamically stable patients and consistent of adequate collection drainage, enteral hyperalimentation, and antibiotic therapy [4].

The aim of this study is to evaluate the safety and efficacy of computed tomography (CT)-guided percutaneous drainage of post-laparoscopic sleeve gastrectomy gastric leaks.

\section{Patients and Methods}

\subsection{Study Design}

We performed a retrospective review of all patients that underwent percutaneous CT-guided drainage of post-laparoscopic gastric leaks due to sleeve gastrectomies from 2011 to 2018 at King Khalid University Hospital, which is a center of excellence for bariatric surgery. All percutaneous CT drainage was performed by an interventional radiologist. The electronic charts of patients were reviewed for complications, success rates, leak healing, and the need for surgery.

\subsection{Inclusion and Exclusion Criteria}

We included all patients with post-laparoscopic gastric leaks that underwent percutaneous CT-guided drainage. We excluded patients with gastric leaks due to other bariatric surgeries and patients without follow-up data.

\subsection{Percutaneous CT-Guided Drainage Technique}

\subsubsection{Preprocedural Assessment}

All patients should undergo CT to confirm gastric leaks. The complete blood count $(\mathrm{CBC})$ and coagulation profile should exhibit acceptable numbers (international normalized ratio (INR) $<1.5$, activated partial thromboplastin time $(\mathrm{aPTT})<55 \mathrm{~s}$, and platelets $>50,000 / \mathrm{lL})$; otherwise, it is mandatory to correct the coagulation profile before the procedure. All anticoagulant medication should be discontinued for an appropriate period of time before the procedure. Fasting for at least 6 hours before the procedure is also required.

\subsubsection{Procedural Technique}

All procedures were performed under CT guidance. First, a CT scan of the upper abdomen was performed, and an area of skin was subsequently marked to indicate the appropriate approach. Next, under sterile technique and CT guidance, an 18-gauge $15-20 \mathrm{~cm}$ chiba needle was advanced into the collection with in- 
termittent CT scanning. Once the needle tip was in the collection, the stylet was removed and the sample was aspirated. Stiff guide wire was advanced into the collection cavity, and the needle was removed. A cone dilator was used to dilate the tract. Finally, a $10-14 \mathrm{Fr}$ drain was inserted so that its tip was as near as possible to the leak site and connected to an external drainage bag (Figure 1).

\subsubsection{Procedural Anesthesia}

Local anesthesia with $1 \%$ lidocaine was administered to superficial and deep tissue along the trajectory tract of drainage. Conscious sedation was administered to the patient with intravenous midazolam and fentanyl, which was tailored to the patient's needs and administered in interval increments starting with low doses.

\subsubsection{Patient Monitoring}

Patient vital signs were continuously monitored during the procedure. Oxygen was supplemented via a nasal canula when oxygen levels fell below $93 \%$.

\subsection{Follow up Imaging}

All patients underwent upper gastrointestinal contrast examinations using water soluble contrast before removal of drain. Drain was removed if no contrast leak was seen (Figure 2).

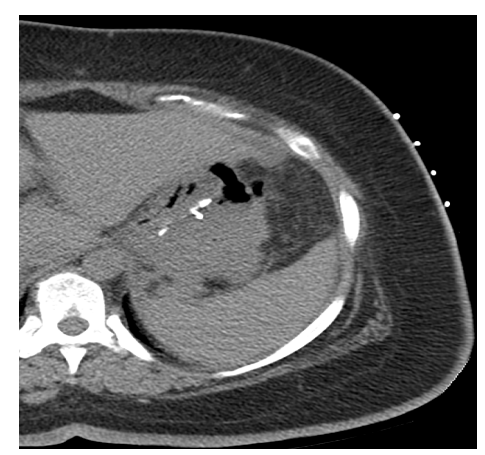

(a)

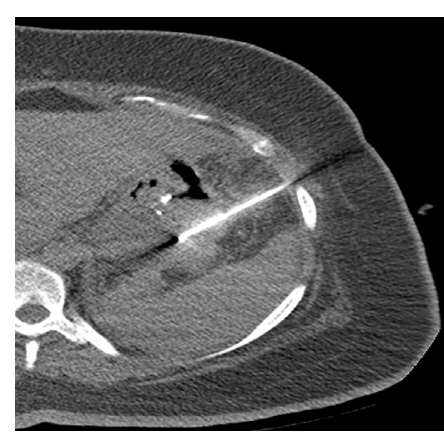

(b)

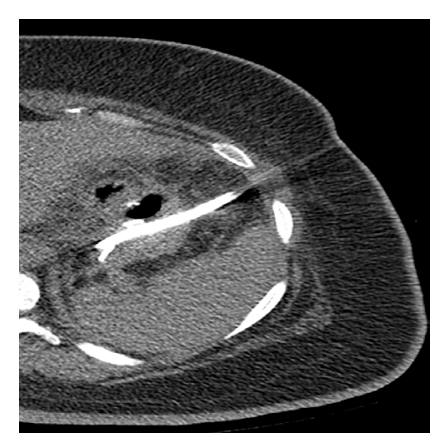

(c)

Figure 1. CT guided drain insertion technique. (a) pre procedural CT scan image with skin marker at the skin entry point; (b) CT scan image with needle inside the perigastric collection; (c) CT scan image with pigtail drain inserted with its tip near leak site. 


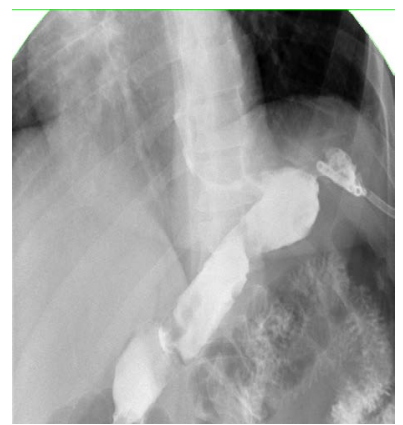

(a)

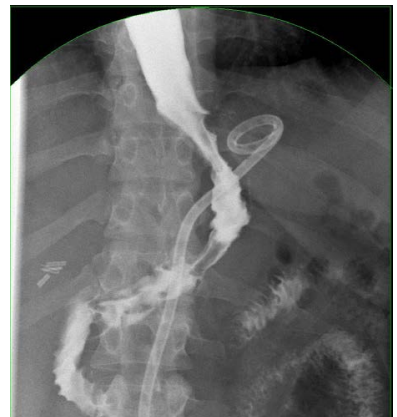

(b)

Figure 2. Upper gastrointestinal contrast study. (a) Upper gastrointestinal contrast study image shows contrast leak at gastroesophageal junction that drains into the pigtail draining tube; (b) Upper gastrointestinal contrast study image shows no contrast leak. Pigtail draining tube is seen with its tip near gastroesophageal junction.

\section{Results}

In total, 78 patients were included ( 44 men and 34 women with an average age of $34.6 \pm 10.5$ years and a body mass index (BMI) of $45 \mathrm{~kg} / \mathrm{m}^{2} \pm 3.2$ ). The success rate of drain insertion was $97.5 \%$ (76/78); the drain was not inserted in two patients $(2.5 \%)$ because the overlying colon prevented safe access. Three approaches were used for drainage: left lateral (53/76), paramidline (19/76), and transhepatic (4/76) (Figure 3).

All patients that underwent drain insertion exhibited early clinical and laboratory improvement, and none required emergency surgery. However, six patients exhibited non-healing leak sites/fistulas and underwent revisional surgery after 3 - 5 months.

One patient developed post-operative hemorrhage due to arterial injury and was managed successfully with transcatheter arterial coil embolization; as such, no surgery was required (Figure 4).

No gastric cutaneous fistulas were observed in the tract of percutaneous drainage. All patients underwent endoluminal stent placement and received antimicrobial therapy and nutritional support. The average indwelling drainage time was 70 days ranging from 42 - 94 days. All patients underwent negative upper gastrointestinal contrast study before drain removal.

\section{Discussion}

Image-guided percutaneous abscess drainage is a well-established and widely used technique for managing patients with localized intra-abdominal fluid collections, which has been proven to be safe and effective [7] [8] [9]. CT is the preferred imaging modality for drainage of deeper intra-abdominal collections [7]. Compared with surgical management, gastrointestinal anastomotic leaks managed by percutaneous image-guided drainage are associated with lower hospital costs and shorter hospital stays [10]. Furthermore, the stomach and the liver may be traversed to allow treatment of an epigastric collection unreachable by other routes [11]. 


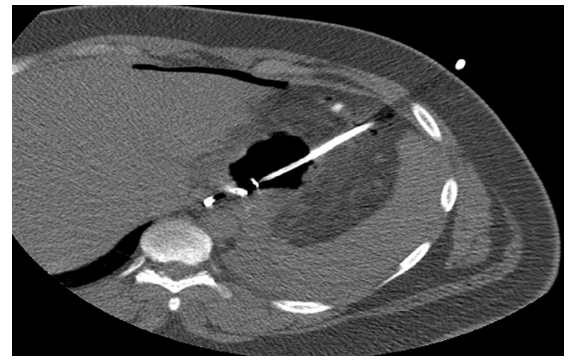

(a)

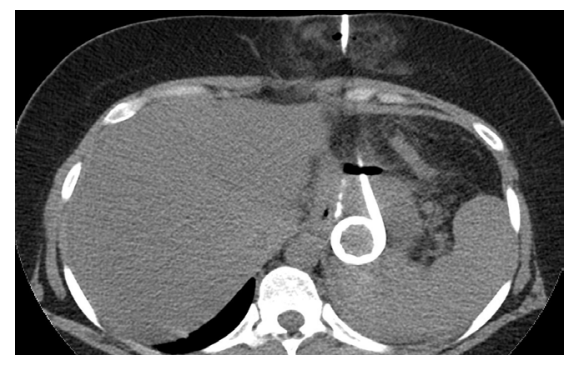

(b)

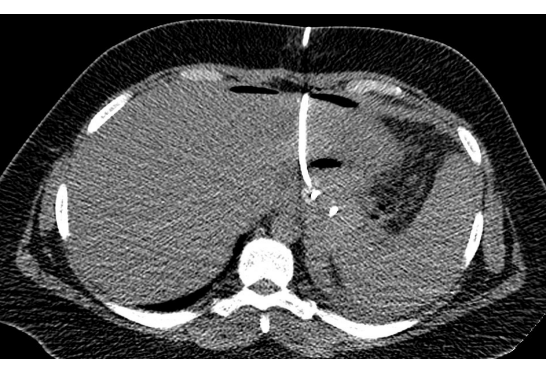

(c)

Figure 3. Three different drainage approaches. (a) left upper abdominal approach; (b) left para midline approach; (c) trans-hepatic approach.

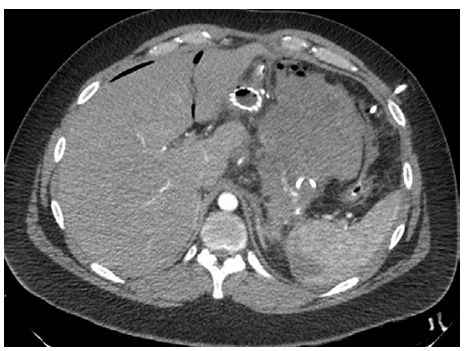

(a)

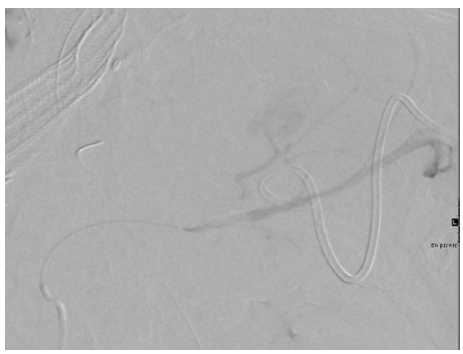

(b)

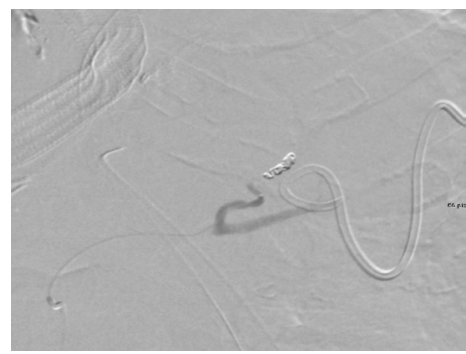

(c)

Figure 4. Post CT guided drainage hemorrhage. (a) CT scan image shows post drainage large hematoma with active contrast extravasation; (b) selective splenic angiogram shows active contrast extravasation from splenic artery branch; (c) post coli embolization selective angiogram shows successful embolization.

Simple drainage alone or combined with stent placement is a safe and effective technique for gastric leak management after sleeve gastrectomy in hemodynamically stable patients. Minimally invasive management exhibits low complication rates and minor discomfort for patients and prevents the need for more invasive procedures or total gastrectomy [6] [12] [13]. However, in cases of chronic fistulas 
that persist for more than 3 months despite adequate drainage, endoluminal therapy, and nutritional support, reoperation is the only curative option [13]. To the best of my knowledge, only two papers have addressed percutaneous image-guided drainage for gastric leaks due to laparoscopic sleeve gastrectomy. Kelogrigoris et al. reported 21 patients who underwent CT-guided drainage with a technical success rate of $100 \%$ and a clinical success rate of $86 \%$. Palermo et al. reported 37 patients of whom $87.5 \%$ exhibited clinical success and $13.5 \%$ required reoperation [14] [15].

In our study, CT-guided percutaneous drainage of gastric leaks due to sleeve gastrectomy exhibited a technical and early clinical success rate of $97.5 \%$. However, $8 \%$ of the patients $(6 / 76)$ required revisional surgery due to persistent gastric leaks/fistulas. We have also described multiple percutaneous drainage approaches for this particular location at left sub diaphragmatic area (left para-midline, left upper abdomen, and transhepatic). We always made the entry point of the pigtail drainage catheter as caudally as possible to avoid diaphragm injury and pleural transgression and its tip as near as possible to the leak site, which was mostly at the gastroesophageal junction (angle of His). Furthermore, we have reported the largest number of patients that underwent conservative management. Pequignot et al. reported that the disadvantage of percutaneous drainage is the creation and potential long-term presence of an external fistula [16]; however, we have not encountered this complication in our case series. On the contrary, we encountered a major complication with a vascular injury to the branch of the splenic artery, which required emergency transcatheter coil embolization of the bleeding artery. The limitation of our study is its retrospective nature. Additionally, all patients underwent endoluminal stent placement, which contributed to the therapeutic success and cannot be separated from the success of percutaneous drainage in our study.

\section{Conclusion}

Percutaneous CT-guided drainage of post-sleeve gastrectomy gastric leaks is a safe, effective, and minimally invasive alternative to surgery. This procedure, along with other conservative measures including endoluminal stent placement as well as antimicrobial and nutritional support, cures most gastric leaks due to sleeve gastrectomy and helps avoid revisional surgery.

\section{Acknowledgements}

I would like to thank Editage (http://www.editage.com/) for English language editing.

\section{Conflicts of Interest}

The author declares that he has no conflicts of interest concerning this article. 


\section{References}

[1] Currò, G., Piscitelli, G., Lazzara, C., Komaei, I., Fortugno, A., Pinto, G., Guccione, F., Cogliandolo, A., Dattola, A., Latteri, S. and Navarra, G. (2017) Laparoscopic Sleeve Gastrectomy for Morbid Obesity: Role of Intraluminal and Intraperitoneal Postoperative Drainage. Il Giornale di Chirurgia, 38, 181-184. https://doi.org/10.11138/gchir/2017.38.4.181

[2] Rosenthal, R.J., Diaz ,A.A., Arvidsson, D., Baker, R.S., Basso, N., Bellanger, D., Boza, C., El Mourad, H., France, M., Gagner, M., Galvao-Neto, M., Higa, K.D., Himpens, J., Hutchinson, C.M., Jacobs, M., Jorgensen, J.O., Jossart, G., Lakdawala, M., Nguyen, N.T., Nocca, D., Prager, G., Pomp, A., Ramos, A.C., Rosenthal, R.J., Shah, S., Vix, M., Wittgrove, A. and Zundel, N. (2012) International Sleeve Gastrectomy Expert Panel Consensus Statement: Best Practice Guidelines Based on Experience of > 12000 Cases. Surgery for Obesity and Related Diseases, 8, 8-19.

https://doi.org/10.1016/j.soard.2011.10.019

[3] Tan, J.T., Kariyawasam, S., Wijeratne, T. and Chandraratna, H.S. (2010) Diagnosis and Management of Gastric Leaks after Laparoscopic Sleeve Gastrectomy for Morbid Obesity. Obesity Surgery, 20, 403-409.

https://doi.org/10.1007/s11695-009-0020-7

[4] Donatelli, G., Dumont, J.L., Cereatti, F., Ferretti, S., Vergeau, B.M., Tuszynski, T., Pourcher, G., Tranchart, H., Mariani, P., Meduri, A., Catheline, J.M., Dagher, I., Fiocca, F., Marmuse, J.P. and Meduri, B. (2015) Treatment of Leaks Following Sleeve Gastrectomy by Endoscopic Internal Drainage (EID). Obesity Surgery, 25, 1293-1301. https://doi.org/10.1007/s11695-015-1675-X

[5] Abou Rached, A., Basile, M. and El Masri, H. (2014) Gastric Leaks Post Sleeve Gastrectomy: Review of Its Prevention and Management. World Journal of Gastroenterology, 20, 13904-13910. https://doi.org/10.3748/wjg.v20.i38.13904

[6] Casella, G., Soricelli, E., Rizzello, M., Trentino, P., Fiocca, F., Fantini, A., Salvatori, F.M. and Basso, N. (2009) Nonsurgical Treatment of Staple Line Leaks after Laparoscopic Sleeve Gastrectomy. Obesity Surgery, 19, 821-826. https://doi.org/10.1007/s11695-009-9840-8

[7] Jaffe, T.A. and Nelson, R.C. (2016) Image-Guided Percutaneous Drainage: A Review. Abdominal Radiology, 41, 629-636. https://doi.org/10.1007/s00261-016-0649-3

[8] Okita, Y., Mohri, Y., Kobayashi, M., Araki, T., Tanaka, K., Inoue, Y., Uchida, K., Yamakado, K., Takeda, K. and Kusunoki, M. (2013) Factors Influencing the Outcome of Image-Guided Percutaneous Drainage of Intra-Abdominal Abscess after Gastrointestinal Surgery. Surgery Today, 43, 1095-1102. https://doi.org/10.1007/s00595-013-0504-x

[9] Men, S., Akhan, O. and Koroglu, M. (2002) Percutaneous Drainage of Abdominal Abscess. European Journal of Radiology, 43, 204-218. https://doi.org/10.1016/S0720-048X(02)00156-0

[10] Burke, L.M.B, Bashir, M.R., Gardner, C.S., Parsee, A.A., Marin, D., Vermess, D., Bhattacharya, S.D., Thacker, J.K. and Jaffe, T.A. (2015) Image-Guided Percutaneous Drainage vs. Surgical Repair of Gastrointestinal Anastomotic Leaks: Is There a Difference in Hospital Course or Hospitalization Cost? Abdominal Imaging, 40, 1279-1284. https://doi.org/10.1007/s00261-014-0265-z

[11] Maher, M.M., Gervais, D.A., Kalra, M.K., Lucey, B., Sahani, D.V., Arellano, R., Hahn, P.F. and Mueller, P.R. (2004) The Inaccessible or Undrainable Abscess: How to Drain It. Radiographics, 24, 717-735. https://doi.org/10.1148/rg.243035100 
[12] Deitel, M., Gagner, M., Erickson, A.L. and Crosby, R.D. (2011) Third International Summit: Current Status of Sleeve Gastrectomy. Surgery for Obesity and Related Diseases, 7, 749-759. https://doi.org/10.1016/j.soard.2011.07.017

[13] Garofalo, F., Pescarus, R., Denis, R., Atlas, H., Garneau, P., Philie, M. and Sayegh, K. (2018) Laparoscopic Sleeve Gastrectomy: A Radiological Guide to Common Postsurgical Failure. Canadian Association of Radiologists Journal, 69, 184-196. https://doi.org/10.1016/j.carj.2017.10.004

[14] Kelogrigoris, M., Sotiropoulou, E., Stathopoulos, K., Georgiadou, V., Philippousis, P. and Thanos, L. (2011) CT-Guided Percutaneous Drainage of Infected Collections Due to Gastric Leak after Sleeve Gastrectomy for Morbid Obesity: Initial Experience. Cardiovascular and Interventional Radiology, 34, 585-589. https://doi.org/10.1007/s00270-010-9928-5

[15] Palermo, M., Davrieux, C.F., Acquafresca, P.A., Gagner, M., Serra, E., Hougthon, E.J., Finger, C. and Giménez, M.E. (2019) Percutaneous Image-Guided Abdominal Interventions for Leaks and Fistulas Following Sleeve Gastrectomy and Roux-en-Y Gastric Bypass. Obesity Surgery, 29, 2051-2058.

https://doi.org/10.1007/s11695-019-03824-5

[16] Pequignot, A., Fuks, D., Verhaeghe, P., Dhahri, A., Brehant, O., Bartoli, E., Delcenserie, R., Yzet, T. and Regimbeau, J.M. (2012) Is There a Place for Pigtail Drains in the Management of Gastric Leaks after Laparoscopic Sleeve Gastrectomy? Obesity Surgery, 22, 712-720. https://doi.org/10.1007/s11695-012-0597-0 